\title{
Distribución temporal del fitoplancton y epiliton en el río Piedra Blanca (Córdoba, Argentina).
}

\author{
María Elisa Luque ${ }^{1} \&$ Ana Luján Martínez de Fabricius ${ }^{1}$ \\ ${ }^{1}$ Departamento de Ciencias Naturales, Facultad de Ciencias Exactas Físico-Químicas y Naturales. UNRC. Ruta \\ 36 Km 601 (5800) Río Cuarto, Córdoba, Argentina. E-mail: mluque@exa.unrc.edu.ar y alujan@exa.unrc.edu.ar
}

\begin{abstract}
RESUMEN
El objetivo de esta investigación es analizar los patrones temporales en la composición y abundancia de las comunidades del fitoplancton y epiliton en el río Piedra Blanca, así como la influencia de los factores ambientales sobre los patrones biológicos durante un ciclo anual. Para este estudio se recolectaron mensualmente muestras cualitativas y cuantitativas de algas en transporte y las adheridas a substrato rocoso, desde junio de 1988 a junio de 1989, en un punto del río mencionado. Se registraron simultáneamente parámetros físicos e hidráulicos. En las muestras planctónicas se determinaron 221 taxa y en el epiliton 170 taxa. La densidad del fitoplancton osciló entre $2 \mathrm{org} / \mathrm{ml} \mathrm{y} 425 \mathrm{org} / \mathrm{ml}$ y la densidad del epiliton varió entre $8240 \mathrm{org} / \mathrm{cm}^{2}$ y 239 $644 \mathrm{org} / \mathrm{cm}^{2}$. El método de clasificación divisivo Twinspan mostró las diferencias cuantitativas existentes en las comunidades durante el ciclo anual. La estructura y dinámica de los organismos del plancton y de las formas adheridas estuvieron principalmente controladas por factores físicos. El pH, conductividad y sólidos disueltos permitieron explicar las variaciones de riqueza de especies y densidad en ambas comunidades.
\end{abstract}

Palabras clave: densidad - fitoplancton- epiliton - Bacillariophyceae - Chlorophyceae - comunidad.

\begin{abstract}
We analyzed the seasonal patterns in the composition and abundance of the planktonic and epilithic communities of the River Piedra Blanca (Córdoba, Argentin) and the influence of environmental factors on the biological patterns during one annual cycle. Qualitative and quantitative samples of phytoplankton and epilithon were taken at one station between June 1988 and June 1989. Physical and hydrological variables were measured concurrently. 221 algae taxa were recorded in the plankton and 170 in the epilithon. The density of the phytoplankton was 2- $425 \mathrm{org} / \mathrm{ml}$, while the density of the epilithon was 8240$239644 \mathrm{org} / \mathrm{cm}^{2}$. Twinspan showed seasonal quantitative differences in the phytoplankton and epilithon. The structure and dynamics of the plankton and epilithon were mainly controlled by physical factors. The pH, conductivity and dissolved solids concentration could explain the variations in the number of species and density of organisms in both communities.
\end{abstract}

Keywords: density, phytoplankton, epilithon, Bacillariophyceae, Chlorophyceae, community.

\section{INTRODUCCIÓN}

El estudio de la estructura y dinámica de la comunidad algal en ríos es complejo debido a los múltiples factores que covarían en el sistema (Rout \& Gaur, 1994). Las comunidades algales son el producto de la interacción de variables geomorfológicas, hidrológicas y bióticas que operan en una escala de amplio rango (Sinsabaugh et al., 1991). El régimen hidrológico ejerce un importante control sobre la biota y es el principal determinante de las variaciones en la abundancia y dominancia de las especies en distintos sitios dentro de un río o en un mismo punto año a año (Allan, 1995; Reynold, 1992). Las asociaciones algales son el resultado de la totalidad de condiciones ambientales, pudiendo así reflejar la estructura y el funcionamiento del río como un ecosistema (Sabater et al., 1988).

En Argentina, la mayoría de los trabajos versan sobre la comunidad fitoplanctónica y son menos los referidos al epiliton. Tal es el caso del estudio de los grandes ríos como el Paraná y Paraguay (García de Emiliani, 1990; Zalocar de 
Domitrovic, 1992; Anselmi de Manavella \& García de Emiliani, 1995). En la provincia de Buenos Aires se han efectuado estudios ecológicos sobre fitoplancton en los ríos Salado y Samborombón (Solari, 1995; O'Farrell, 1993) y en ríos contaminados (Loez \& Salibian, 1990; Del Giorgio et al., 1991; Conforti et al., 1995). Investigaciones en ríos y arroyos de la provincia de Córdoba aportan al conocimiento algal a través de estudios bioecológicos y florísticos iniciados en la cuenca del río Tercero (Martínez de Fabricius, 1986; Martínez de Fabricius et al., 1988; Martínez de Fabricius \& Corigliano, 1989; Martínez de Fabricius \& Gari, 1989, 1991, 1996). Se incorporan, más recientemente, estudios en la cuenca del río Cuarto, desde los arroyos tributarios en la cabecera de la cuenca hasta su derrame en los bañados del Saladillo (Luque et al., 1994, Corigliano et al., 1994;
Martínez de Fabricius, 1995; Luque et al., 1997; Luque \& Martínez de Fabricius, 2000).

El objetivo de esta investigación es analizar los patrones temporales en la composición y abundancia de las comunidades de algas en transporte, en el compartimiento planctónico, y de algas del epiliton en el río Piedra Blanca, así como la influencia de los factores ambientales sobre los patrones biológicos durante un ciclo anual.

\section{DESCRIPCIÓN DEL RÍO PIEDRA BLANCA}

El río Piedra Blanca se ubica en el Departamento de Río Cuarto, entre los $32^{\circ} 54^{\prime} \mathrm{S}$ y $64^{\circ}$ 50 'O, a una altitud de $618 \mathrm{msnm}$. Es uno de los ríos más importantes de la provincia de Córdoba, junto con el río Tercero integran la cuenca exorreica del Carcaraña.

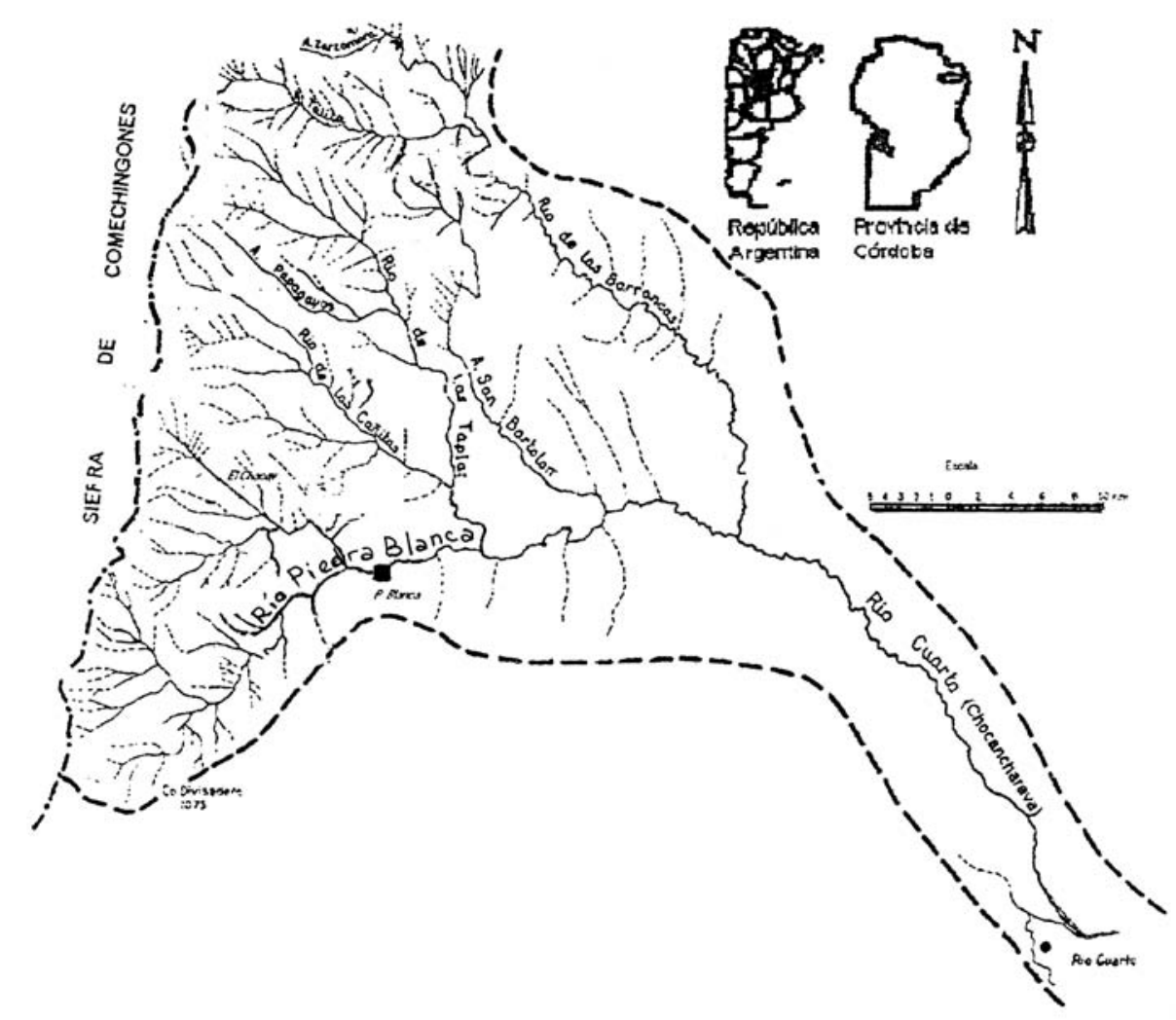

Figura 1. Cuenca hidrográfica activa del río Cuarto. Localidad de muestreo en el río Piedra Blanca (Córdoba, Argentina). River Cuarto active hydrographic basin. Sampling sites in the river Piedra Blanca (Córdoba, Argentina) 
El río Piedra Blanca, según Strahler (1981) de $5^{\circ}$ orden, posee una longitud de $85 \mathrm{~km}$, un ancho medio de $15 \mathrm{~m}$, una profundidad que oscila entre 0.5 a $0.6 \mathrm{~m}$, a excepción de las épocas de crecientes, donde suele alcanzar profundidades medias de $1 \mathrm{~m}$. Este curso posee un régimen hídrico de tipo pluvial, con un caudal anual promedio de $3524 \mathrm{~m}^{3 /} \mathrm{s}$, en el sustrato predominan bloques y guijarros, con tramos muy turbulentos o rabiones, que alternan con los remansos.

\section{MATERIAL Y MÉTODOS}

Se recolectaron un total de 39 muestras, 26 corresponden al plancton y 13 al epiliton, con una frecuencia mensual durante el período comprendido entre junio de 1988 a junio de 1989 en una localidad del río Piedra Blanca, situada $8 \mathrm{~km}$ aguas arriba de la confluencia de los ríos Piedra Blanca y Las Cañitas y distante unos $70 \mathrm{~km}$ de la ciudad de Río Cuarto (Fig. 1 y Tabla 1).

Las muestras de fitoplancton se obtuvieron por filtración de 100 litros de agua a través de una red de plancton de $25 \mu \mathrm{m}$ de diámetro de

Tabla 1. Programa de muestreo realizado en el río Piedra Blanca (Córdoba, Argentina), durante el período Junio 1988-Junio 1989. Sampling programme in the river Piedra Blanca (Córdoba, Argentina) during June 1988-June 1989.

\begin{tabular}{|c|c|c|c|}
\hline \multirow{2}{*}{ FECHA } & \multicolumn{3}{|c|}{ COMUNIDAD } \\
\hline & \multicolumn{2}{|c|}{ FITOPLANCTON } & \multirow{2}{*}{$\begin{array}{l}\text { EPILITICA } \\
\text { Cuantitativa }\end{array}$} \\
\hline & Cualitativa & Cuantitativa & \\
\hline $22-06-1988$ & 543 & 544 & 545 \\
\hline $26-07-1988$ & 557 & 558 & 559 \\
\hline $23-08-1988$ & 575 & 576 & 577 \\
\hline 26-09-1988 & 618 & 615 & 616 \\
\hline $27-10-1988$ & 629 & 631 & 632 \\
\hline 24-11-1988 & 656 & 653 & 655 \\
\hline $20-12-1988$ & 663 & 662 & 665 \\
\hline 30-01-1989 & 682 & 681 & 696 \\
\hline 22-02-1989 & 697 & 698 & 699 \\
\hline 31-03-1989 & 730 & 729 & 732 \\
\hline 26-04-1989 & 782 & 783 & 784 \\
\hline 24-05-1989 & 801 & 803 & 804 \\
\hline 29-06-1989 & 815 & 816 & 817 \\
\hline
\end{tabular}

poro. Las epilíticas fueron obtenidas por raspado de una superficie promedio de $100 \mathrm{~cm}^{2}$, lavadas con agua destilada estéril. Para ello se recolectaron pequeñas rocas del fondo según un muestreo estratificado al azar, donde el sitio de muestreo se divide en estratos homogéneos y las muestras obtenidas son colectadas al azar de cada estrato (Venrick, 1995). Simultáneamente se registraron los siguientes parámetros físicos e hidráulicos: temperatura y $\mathrm{pH}$ mediante el uso de P. Altronix M-206 y la conductividad con sensor Altronix CT2, sólidos disueltos, velocidad de corriente (mediante el uso de un objeto desplazado por el agua: flotador) y caudal.

Las muestras se incorporaron al Herbario del Departamento de Ciencias Naturales de la UNRC, con la sigla RCC. Para el análisis taxonómico se utilizó bibliografía específica para cada grupo y/o sección en particular: Desikachary (1959), Prescott (1962), Archibald (1983), Patrick \& Reimer (1966, 1975), Germain (1981), Krammer \& Lange-Bertalot (1986, 1988, 1991a, 1991b). El total de las especies determinadas se ordenaron según Bourrelly $(1981,1985$, 1990) para las Cyanophyceae, Chlorophyceae, Dinophyceae y Euglenophyceae y según Simonsen (1979) para las Bacillariophyceae.

Los recuentos se realizaron por transectas según el método propuesto por Villafañe \& Reid (1995). Se predeterminaron tres transectas horizontales equidistantes evitando los efectos de bordes. Se utilizó un portaobjetos y un cubreobjetos de 24 x $50 \mathrm{~mm}$, en el portaobjetos se sedimentó una alícuota de $0.3 \mathrm{ml}$, esto permitió la observación del material con un aumento de $400 \mathrm{X}$. Se contaron más de una cámara hasta obtener un promedio de individuos (unicelulares, cenobios, colonias o filamentos) constante, el cual se logró con cinco cámaras en las muestras de fitoplancton y tres cámaras en las muestras del epiliton. Para los organismos filamentosos, Lyngbya, Oscillatoria, se consideró como unidad una longitud equivalente al largo de un organismo unicelular frecuente como Synedra y/o Fragilaria.

Se calculó el índice de diversidad: H (Shannon \& Weaver, 1963) y el componente de equitabilidad: E. Mediante el método de clasificación divi- 


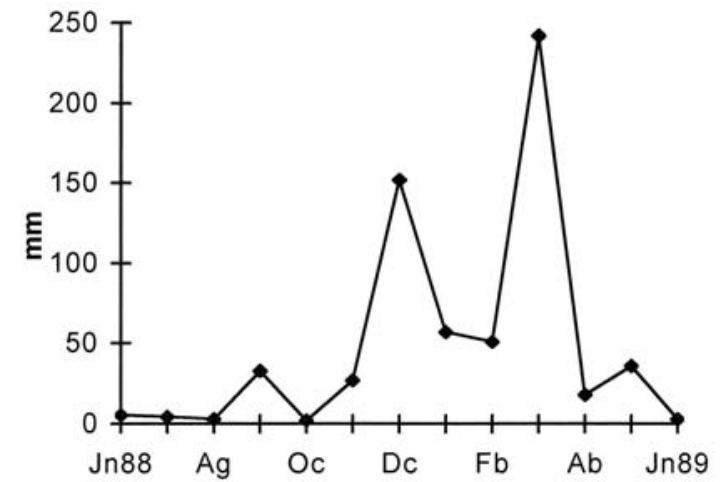

Figura 2. Promedios mensuales de precipitaciones registradas en el área de estudio (río Piedra Blanca, Córdoba, Argentina) durante el período Junio 1988-Junio 1989. Monthly mean rainfall in the study area (river Piedra Blanca, Córdoba, Argentina) during the period June 1988-June 1989.

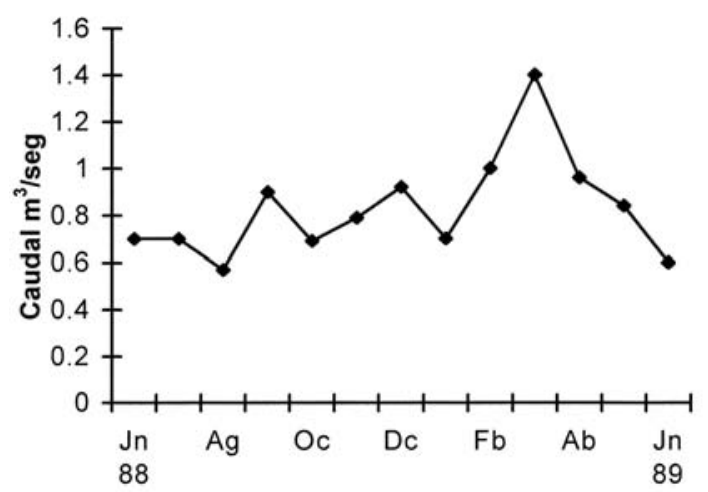

Figura 3. Valores mensuales de caudal del río Piedra Blanca (Córdoba, Argentina), durante el año de muestreo Junio 1988Junio 1989. Monthly mean discharge of river Piedra Blanca (Córdoba, Argentina) during the period June 1988-June 1989.

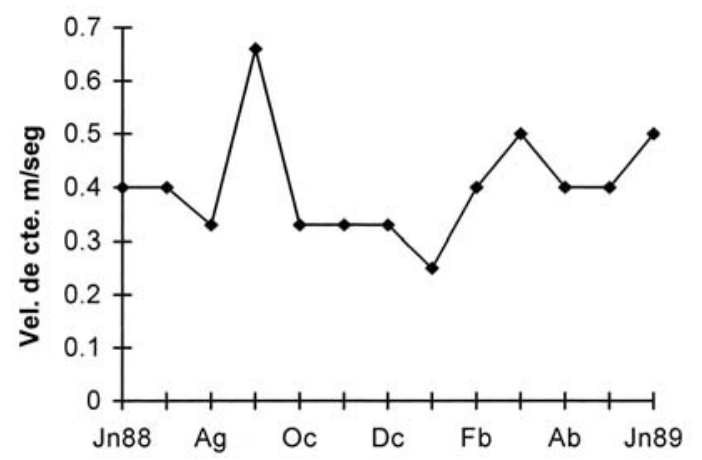

Figura 4. Fluctuaciones de la velocidad de corriente en el río Piedra Blanca (Córdoba, Argentina), durante el año de muestreo Junio 1988-Junio 1989. Variations in water current velocity of river Piedra Blanca (Córdoba, Argentina), during the period June 1988-June 1989. sivo TWINSPAN (Hill, 1979) se comparó el fitoplancton y el epiliton.

Se determinaron los estadísticos descriptivos de las variables fisicas y biológicas, comprobándose su normalidad mediante la prueba de $\chi^{2}$-cuadrado (Sokal \& Rohl, 1979). En caso de distribuciones no normales, los datos fueron transformados logarítmicamente (logaritmo decimal). La relación entre variables se analizó a partir de los coeficientes de correlación lineal simple ( $\mathrm{r}$ de Pearson) y los parámetros de las ecuaciones de regresión lineal. Para el análisis de la influencia de los parámetros ambientales sobre los descriptores biológicos se utilizó el análisis de correlación múltiple.

\section{RESULTADOS}

\section{Parámetros hidrológicos y físicos}

Dado que el régimen hídrico es de tipo pluvial, las variaciones estacionales del caudal están determinadas por las precipitaciones, las que oscilaron entre $242 \mathrm{~mm}$ en marzo y $0 \mathrm{~mm}$ en junio de 1989. El caudal varió entre $0.57 \mathrm{~m}^{3} / \mathrm{s}$ y $1.4 \mathrm{~m}^{3} / \mathrm{s}$. La velocidad de corriente fluctuó entre $0.25 \mathrm{~m} / \mathrm{s}$ y $0.66 \mathrm{~m} / \mathrm{s}$ (Figs. 2,3 y 4 ).

Durante el año de muestreo la temperatura varió entre $6^{\circ} \mathrm{C}$ y $28^{\circ} \mathrm{C}$. El pH osciló entre 6.5 y 8.5. La conductividad registró valores comprendidos entre $92 \mu \mathrm{S} / \mathrm{cm}$ y $278 \mu \mathrm{S} / \mathrm{cm}$ (Figs. 5, 6 y 7).

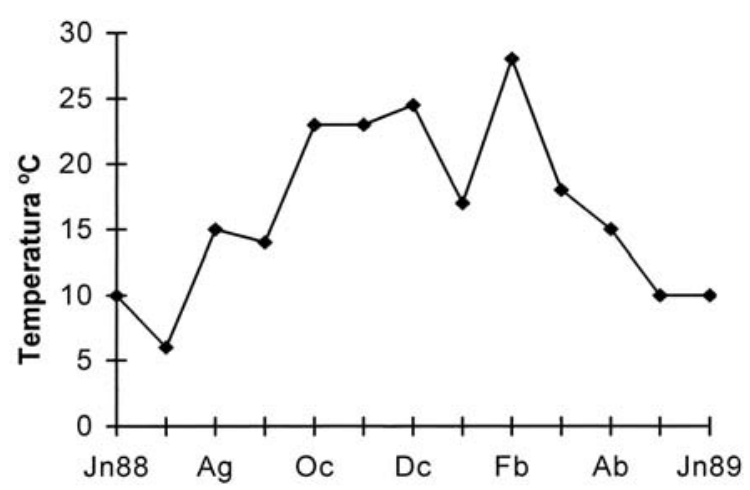

Figura 5. Valores mensuales de la temperatura en el río Piedra Blanca (Córdoba, Argentina), durante el período Junio 1988Junio 1989. Monthly mean temperature of river Piedra Blanca (Córdoba, Argentina), during the period June 1988-June 1989. 


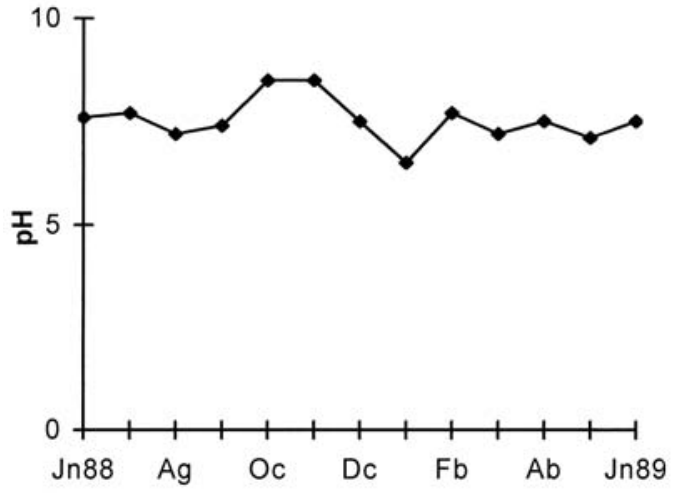

Figura 6. Valores mensuales de $\mathrm{pH}$ en el río Piedra Blanca, durante el período 1988-1989. Monthly values of the River Piedra Blanca $\mathrm{pH}$, during the 1988-1989 period.

\section{Aspectos biológicos}

Del análisis taxonómico de las comunidades fitoplanctónica y epilítica se determinaron un total de 261 taxa. De ellas, 196 son Bacillariophyceae, 48 Chlorophyceae, 13 Cyanophyceae, 2 Dinophyceae y 2 Euglenophyceae (Tabla 2).

\section{Organismos en el plancton:}

La densidad de los organismos en fitoplancton osciló entre $2 \mathrm{org} / \mathrm{ml}$ en el mes de junio de 1988 y $425 \mathrm{org} / \mathrm{m}$ en junio de 1989 (Fig. 8). La clase Bacillariophyceae predominó en todo el período de estudio, su densidad fluctúo entre $2 \mathrm{org} / \mathrm{ml}$ en

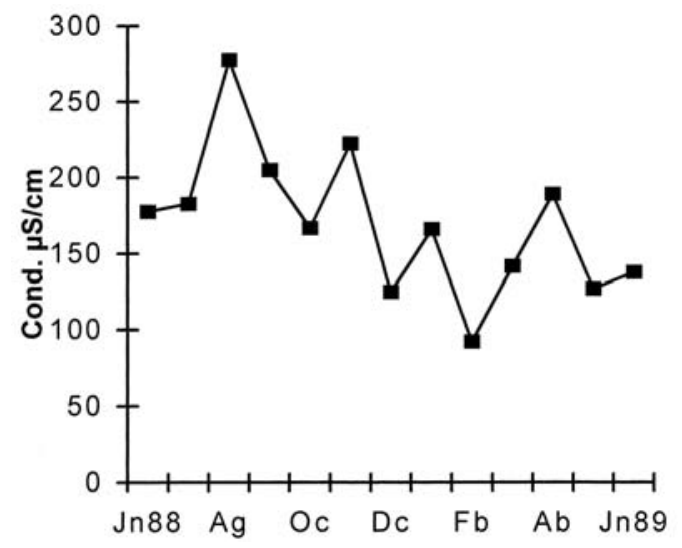

Figura 7. Valores mensuales de conductividad en el río Piedra Blanca (Córdoba, Argentina) durante el período Junio 1988Junio 1989. Monthly mean conductivity of river Piedra Blanca (Córdoba, Argentina), during the period June 1988-June 1989.

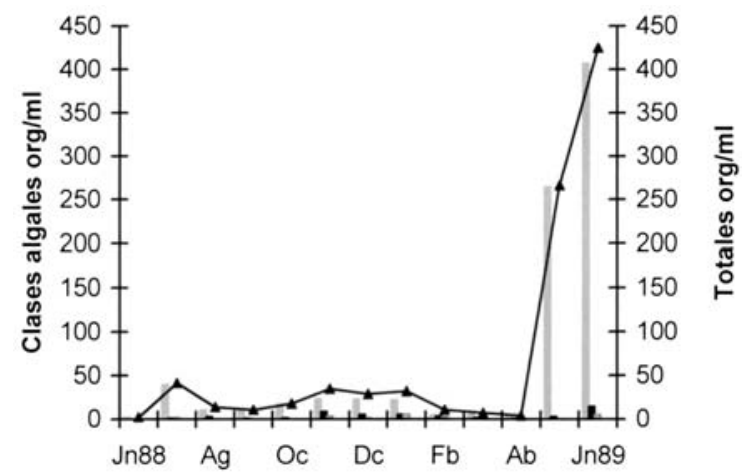

Figura 8. Variación de la densidad total y por clases algales de la comunidad fitoplanctónica en el río Piedra Blanca (Córdoba, Argentina) durante el período Junio 1988-Junio 1989. Variation in density of total phytoplankton and of each algal group in river Piedra Blanca (Córdoba, Argentina) during the period June 1988-June 1989.

el mes de junio de 1988 y 407 org/ml en junio de 1989. Las especies Cocconeis placentula var. lineata, C. placentula var. euglypta, Melosira varians y Reimeria uniseriata fueron más abundantes durante los meses de mayo y junio de 1989. La concentración de las Chlorophyceae osciló entre $0.13 \mathrm{org} / \mathrm{ml}$ y $14.3 \mathrm{org} / \mathrm{ml}$. Cladophora glomerata, Dictyosphaerium pulchellum, Monoraphidium sp., Pediastrum boryanum y Scenedesmus ecornis, presentaron su mayor densidad en los meses de verano. Especies de Cyanophyceae registraron bajas densidades, las que fluctuaron entre $0.04 \mathrm{org} / \mathrm{ml}$ y $5 \mathrm{org} / \mathrm{ml}$. La más abundante fue Lyngbya $\mathrm{sp}_{1}$ (Tabla 3 ).

La diversidad fluctuó entre 1.63 bits/org para el mes de setiembre y 3.57 bits/org en el mes de diciembre. La equitabilidad osciló entre 0.52 en setiembre y 0.85 en febrero de 1989 (Tabla 4).

Organismos en el epiliton:

La densidad de los organismos adheridos osciló entre $8240 \mathrm{org} / \mathrm{cm}^{2}$ en el mes de octubre y 239 $644 \mathrm{org} / \mathrm{cm}^{2}$ en setiembre del mismo año (Fig. 9).

La clase Bacillariophyceae fue la más abundante con valores comprendidos entre 5769 org/ $\mathrm{cm}^{2}$ en enero y $231457 \mathrm{org} / \mathrm{cm}^{2}$ en setiembre. Las especies Achnanthes minutissima, Cocconeis placentula var. euglypta, C. placentu- 
Tabla 2. Lista taxonómica de algas identificadas en el fitoplancton y epiliton del río Piedra Blanca (Córdoba, Argentina), Junio 1988-Junio 1989. List of phytoplankton and epilython taxa found in the river Piedra Blanca (Córdoba, Argentina) during the period June 1988-June 1989.

\section{Clase CYANOPHYCEAE}

Chroococcus sp.

Merismopedia glauca (Ehrenberg) Naegeli

Synechococcus aeruginosus Naegeli

Anabaena constricta (Szafer) Geitler

Anabaena sp.

Lyngbya limnetica Lemmermann

Lyngbya $\mathrm{sp}_{1}$.

Lyngbya $\mathrm{sp}_{2}$.

Oscillatoria amoena (Kützing) Gomont

O. limosa Agardh

O. minima Gicklhorn

O. nigra Vaucher

O. tenuis Agardh

Oscillatoria sp.

Plectonema sp.

Rivularia sp.

\section{Clase CHLOROPHYCEAE}

Planktonsphaeria gelatinosa G. M. Smith

Tetraedron minimum (A. Braun) Hansgirg

Chlamydomonas sp.

Pandorina morum Bory

Planktosphaeria gelatinosa G. M. Smith

Tetraedron minimum (A. Braun) Hansgirg

Sphaerocystis sp.

Ankistrodesmus falcatus (Corda) Ralfs

Ankistrodesmus fusiformis Corda

Ankistrodesmus spiralis (Turner) Lemmermann

Kirchneriella lunaris (Kirchner) Moebius

Monoraphidium sp.

Oocystis sp.

Selenastrum gracile Reinsch

Selenastrum sp.

Dictyosphaerium pulchellum Wood

D. ehrenbergianum Naegeli

Eutetramorus fottii (Hindák) Kómarek

Coelastrum sp.

Scenedesmus acuminatus (Lagerheim) Chodat

S. acutiformis Schroeder

S. acutus Meyen

S. acutus f. costulatus (Chodat) Uherkovic

S. brevispina (Smith) Chodat

S. denticulatus Lagerheim

S. dimorphus (Turpin) Kützing

S. ecornis (Ralfs) Chodat

S. falcatus f. maximus Uherkovic

S. intermediuus Chodat

S. obliquus (Turpin) Kützing

S. spinosus Chodat
Clase CHLOROPHYCEAE (continuación)

Hydrodictyon reticulatum (L.) Lagerheim

Pediastrum boryanum (Turpin) Meneghini

P. boryanum var. caribeanum Comas

$P$. duplex Meyen

P. duplex var. clathratum Sulek

$P$. simplex Meyen

P. tetras (Ehrenberg) Ralfs

Pediastrum sp.

Microspora sp.

Stigeoclonium stagnatile (Hazen) Collins

Oedogonium sp.

Cladophora glomerata (L.) Kützing

Spirogyra sp.

Zygnema sp.

Closterium acerosum (Schrank) Ehrenberg

Closterium aciculare West

Closterium acutum (Lyngbie) Brébisson

C. dianae Kützing

C. libellula Focke

C. moniliferum (Bory) Ehrenberg

C. parvulum Naegeli

C. subulatum (Kützing) Brébisson

Closterium $\mathrm{sp}_{1}$

Closterium $\mathrm{sp}_{2}$.

Closterium $\mathrm{sp}_{3}$.

Euastrum spinulosum Delponte

E. verrucosum Ehrenberg

Cosmarium botrytis (Bory) Meneghini

C. laeve var. octangulare (Wille) West et West

C. meneghinii Brébisson

C. pseudopyramidatum Lund

C. punctulatum Brébisson

C. reniforme Archer

C. subspeciosum Nordst

C. subspeciosum var. validius Nordstedt

C. turpinii Brébisson

Cosmarium $\mathrm{sp}_{1}$.

Cosmarium $\mathrm{sp}_{2}$.

Penium sp.

Staurastrum brevispinum Brébisson

S. punctulatum Brébisson

Staurodesmus dickiei Lillieroth

\section{Clase EUGLENOPHYCEAE}

Euglena sp.

Phacus sp.

Clase DINOPHYCEAE

Peridinium gatunense Nygaard

Peridinium sp. 
Tabla 2. Continuación. Continuation.

\section{Clase BACILLARIOPHYCEAE}

Aulacoseira granulata (Ehrenberg) Simonsen

A. italica (Ehrenberg) Simonsen

Cyclotella kützingiana Thwaites

C. meneghiniana Kützing

C. striata (Kützing) Grunow

Melosira varians Agardh

Diatoma anceps (Ehrenberg) Kirchner

D. tenue Agardh

Diatoma vulgare Bory

Diatoma sp.

Fragilaria bidens Heiberg

F. capucina var. mesolepta Rabenhorst

F. construens (Ehrenberg) Grunow

F. construens var. venter (Ehrenberg) Grunow

F. crotonensis var. oregona Sovereign

F. elliptica Schumann

F. leptostauron var. dubia (Grunow) Hustedt

F. vaucheriae (Kützing) Petersen

Fragilaria $\mathrm{sp.}$

Meridion circulare (Greville) Agardh

Synedra acus Kützing

S. parasitica (W. Smith) Hustedt

S. rumpens Kützing

S. rumpens var. familiaris (Kützing) Grunow

S. ulna (Nitzsch) Ehrenberg

S. ulna var. oxyrhynchus f. mediocontracta (Forti) Hustedt

S. ulna var. spathulifera Grunow

Eunotia arcus Ehrenberg

E. didyma var. media Hustedt

E. maior (W. Smith) Rabenhorst

E. monodon Ehrenberg

E. pectinalis (Dillwyn) Rabenhorst

Eunotia sp.

Achnanthes clevei Grunow

A. clevei var. rostrata Hustedt

A. coarctata (Brébisson) Grunow

A. conspicua A. Mayer

A. deflexa Reimer

A. delicatula Kützing

A. exigua Grunow

A. exigua var. constricta (Torka) Hustedt

A. inflata (Kützing) Grunow

$A$. helvetica (Hustedt) Lange-Bertalot

A. lanceolata (Brébisson) Grunow

A. lanceolata var. omissa Reimer

A. lanceolata var. rostrata (Oestrup) Hustedt

A. linearis f. curta (W. Smith) Grunow

A. minutissima Kützing

A. rupestris Krasske

Achnanthes $\mathrm{sp}_{1}$.
Achnanthes $\mathrm{sp}_{2}$.

Achnanthes $\mathrm{sp}_{3}$.

Achnanthes $\mathrm{sp}_{4}$.

Achnanthes $\mathrm{sp}_{5}$.

Cocconeis placentula var. euglypta (Ehrenberg) Cleve

C. placentula var. lineata (Ehrenberg) Van Heurck

Amphipleura lindheimerii Grunow

Amphora bullatoides Hohn et Hellerman

$P$. brevicostata Cleve

P. burkii Patrick

P. dactylus Ehrenberg

$P$. divergens W. Smith

P. gibba Ehrenberg

P. gibba var. mesogongyla (Ehrenberg) Hustedt

$P$. maior (Kützing) Rabenhorst

P. mesogongyla Ehrenberg

P. microstauron (Ehrenberg) Cleve

P. microstauron var. brevissonii (Kützing) Mayer

$P$. nobilis (Ehrenberg) Ehrenberg

P. obscura Krasske

P. streptoraphe var. minor Cleve

P. viridis (Nitzsch) Ehrenberg

Pinnularia sp.

Reimeria uniseriata Sala, Guerrero y Ferrario Rhoicosphenia abbreviata (Agardh) Lange-Bertalot Stauroneis phoenicenteron (Nitzsch) Ehrenberg

Epithemia adnata (Kützing) Brébisson

E. argus Kützing

E. sorex Kützing

E. turgida (Ehrenberg) Kützing

Epithemia sp.

Rhopalodia acuminata Krammer

R. brevissonii Krammer

R. constricta (W. Smith) Krammer

R. gibba (Ehrenberg) Müller

$R$. gibba var. ventricosa (Kützing) H. et M. Peragallo

R. musculus (Kützing) O. Müller

$R$. operculata (Ehrenberg) O. Müller

Rhopalodia sp.

Denticula elegans Kützing

D. kuetzingii Grunow

Bacillaria paxillifer (O. Muller) Hendey

Hantzschia amphioxys (Ehrenberg) Grunow

$H$. virgata (Roper) Grunow

H. virgata var. capitellata Hustedt

Nitzschia acicularis (Kützing) Wm. Smith

$N$. acicularis var. closterioides Grunow

N. amphibiodes Hustedt

$N$. calida Grunow

N. capitellata Hustedt

$N$. clausii Hantzsch

$N$. constricta (Kützing) Ralfs 
Tabla 2. Continuación. Continuation.

Clase BACILLARIOPHYCEAE (continuación)

N. dissipata (Kützing) Grunow

$N$. fonticola Grunow

$N$. frustulum (Kützing) Grunow

$N$. hungarica Grunow

$N$. inconspicua Grunow

$N$. lacuum Lange-Bertalot

$N$. levidensis (W. Smith) Grunow

$N$. linearis (Agardh) Smith

$N$. palea (Kützing) W. Smith

N. perminuta (Grunow) M. Peragalio

$N$. prolongata Hustedt

N. pusilla Kützing

$N$. recta Hantzsch

N. scalpelliformis Grunow

$N$. sigma (Kützing) W. Smith

$N$. sinuata var. delognei (Grunow) Lange-Bertalot

$N$. sinuata var. tabellaria (Grunow) Grunow

N. umbonata (Ehrenberg) Lange-Bertalot

$N$. valdecostata Lange-Bertalot et Simonsen

Nitzschia $\mathrm{sp.}$

Cymatopleura solea (Brébisson) W. Smith

Surirella angusta Kützing

S. elegans Ehrenberg

S. linearis W. Smith

S. ovalis Brébisson

S. splendida (Ehrenberg) Kützing

A. pediculus (Kützing) Grunow

A. ovalis var. affinis (Kützing) Van Heurck

A. sabiniana Reimer

A. submontana Hustedt

Amphora sp.

Anomoeoneis sphaerophora (Ehrenberg) Pfitzer

Caloneis bacillum (Grunow) Cleve

Cymbella affinis Kützing

C. amphicephala Naegeli

C. aspera (Ehrenberg) Peragallo

C. cistula (Ehrenberg) Kirchner

C. cistula var. gibbosa Brun

C. cymbiformis Agardh

C. microcephala Grunow

C. minuta Hilse ex Rabenhorst

C. muelleri Hustedt

C. silesiaca Bleisch

C. tumida (Brébisson) Van Heurck

C. turgidula Grunow

Cymbella sp.

Diploneis smithii (Brébisson ex W. Smith) Cleve

D. smithii var. dilatata (M. Peragallo) Boyer

D. ovalis (Hilse) Cleve

Frustulia vulgaris (Thwaites) De Toni

Gomphoneis herculeana (Ehrenberg) Cleve
Gomphonema acuminatum Ehrenberg

G. affine Kützing

G. angustatum (Kützing) Rabenhorst

G. clevei Fricke

$G$. aff. demerarae (Grunow) Frenguelli

G. gracile Ehrenberg

G. intricatum Kützing

G. minutum (Agardh) Agardh

G. olivaceum (Lyngbye) Kützing

G. parvulum (Kützing) Grunow

G. parvulum f. saprophilum Lange-Bertalot et Reichardt

$G$. aff rhombicum Fricke

G. pseudotenellum Lange-Bertalot

G. subclavatum (Grunow) Grunow

G. subclavatum var. mexicanum (Grunow) Patrick

G. truncatum Ehrenberg

G. truncatum var. capitatum (Ehrenberg) Patrick

Gomphonema sp.

Gyrosigma acuminatum (Kützing) Rabenhorst

G. eximium (Thwaites) Boyer

Mastogloia sp.

Navicula capitata var. hungarica

$N$. capitata var. hungarica (Grunow) Ross

N. capitatoradiata Germain

N. cryptocephala Kützing

N. cuspidata (Kützing) Kützing

N. cuspidata var. ambigua (Ehrenberg) Cleve

N. charlatii Peragallo

$N$. decussis Oestrup

N. elginensis (Gregory) Ralfs

$N$. elginensis var. neglecta (Krasske) Patrick

$N$. exigua var. capitata Patrick

$N$. hambergii Hustedt

$N$. kotschyi Grunow

$N$. laevissima Kützing

N. menisculus Schumann

N. menisculus var. upsaliensis Grunow

N. minuscula Grunow

N. mutica Kützing

N. mutica var. cohnii (Hilse) Grunow

$N$. mutica var. undulata (Hilse) Grunow

$N$. notha Wallace

$N$. odiosa Wallace

N. perminuta Grunow

$N$. pupula Kützing

$N$. radiosa Kützing

$N$. radiosa var. parva Wallace

$N$. radiosa var. tenella (Brébisson) Grunow

$N$. rhyncocephala Kützing

$N$. salinarum Grunow

N. tripunctata (O. F. Müller) Bory

$N$. veneta Kützing 
Tabla 2. Continuación. Continuation.

Clase BACILLARIOPHYCEAE (continuación)

$N$. viridula (Kützing) Ehrenberg

$N$. viridula var. linearis Hustedt

Navicula sp.

Neidium iridis (Ehrenberg) Cleve

Pinnularia abaujensis (Pantocsek) Ross
P. abaujensis var. linearis (Hustedt) Patrick

P. acrosphaeria W. Smith

$P$. borealis Ehrenberg

$P$. borealis var. rectangularis Carlson

P. braunii var. amphicephala (A. Mayer) Hustedt

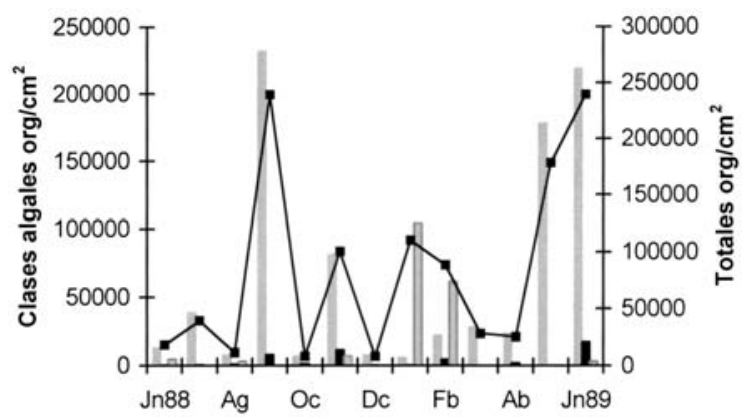

Figura 9. Variación de la densidad total y por clases algales de la comunidad epilítica en el río Piedra Blanca (Córdoba, Argentina) durante el período Junio 1988-Junio 1989. Variation in density of total epilithon and of each algal group in river Piedra Blanca (Córdoba, Argentina) during the period June 1988-June 1989. la var. lineata, Cymbella affinis, Navicula cryptocephala, Nitzschia frustulum y Synedra ulna fueron más abundantes en los meses de setiembre de 1988, y mayo y junio de 1989. El mínimo valor de densidad de las Chlorophyceae se registró en el mes de marzo con $93 \mathrm{org} / \mathrm{cm}^{2}$ y el máximo fue de $17353 \mathrm{org} / \mathrm{cm}^{2}$ en el mes de junio de 1989, Stigeoclonium stagnatile, Monoraphidium sp. y Cladophora glomerata fueron las más abundantes. En la clase Cyanophyceae la mínima densidad se presentó en el mes de diciembre con $28 \mathrm{org} / \mathrm{cm}^{2}$ y la más elevada en el mes de enero con $104343 \mathrm{org} / \mathrm{cm}^{2}$. En esta comunidad Lyngbya sp también fue la más abundante (Tabla 3).

Tabla 3. Composición específica y densidad de la comunidad fitoplanctónica y epilítica del río Piedra Blanca (Córdoba, Argentina) durante el periodo Junio 1988-Junio 1989. Phytoplankton and epilithon species composition and abundance in river Piedra Blanca (Córdoba, Argentina) during the period June 1988-June 1989.

Fitoplancton

\begin{tabular}{lr}
\hline Especies & Den \\
\hline BACILLARIOPHYCEAE & \\
Cocconeis placentula var lineata & 119 \\
Cocconeis placentula var euglypta & 119 \\
Reimeria uniseriata & 47 \\
Melosira varians & 21
\end{tabular}

\section{CHLOROPHYCEAE}

Spirogyra sp.

Dictyosphaerium pulchellum

Pediastrum boryanum

Scenedesmus ecornis

CYANOPHYCEAE

Lyngbya $\mathrm{sp}_{1}$

\section{Epiliton}

Especies Densidad Org $/ \mathrm{cm}^{2}$

\section{BACILLARIOPHYCEAE}

Synedra ulna $\quad 65614$

Cymbella affinis $\quad 429245$

Navicula cryptocephala $\quad 32811$

Cocconeis placentula var lineata $\quad 31453$

Achnanthes minutissima $\quad 29627$

Cocconeis placentula var euglypta $\quad 29083$

Nitzschia frustulum 25087

\section{CHLOROPHYCEAE}

Stigeoclonium stagnatile $\quad 15843$

Monoraphidium sp. $\quad 4225$

Cladophora glomerata 2632

CYANOPHYCEAE

Lyngbya $\mathrm{sp}_{1}$

104342 

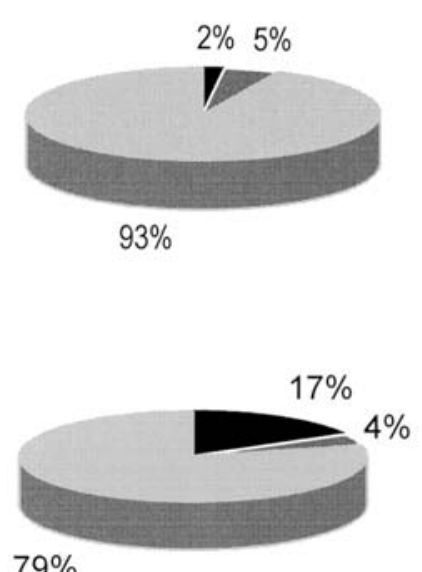

Figura 10. Distribución anual en tantos por ciento de la densidad del fitoplancton y epiliton en el río Piedra Blanca (Córdoba, Argentina) durante el período Junio 1988-Junio 1989. Annual percentage of phytoplankton and epilithon densities in the River Piedra Blanca (Córdoba, Argentina) during the period June 1988-June 1989.

El índice de diversidad varió entre 0.48 bits/cél en enero y 4.15 bits/cél, en el mes de mayo. La equitabilidad presentó un rango de variación entre 0.17 en el mes de enero y 1 en agosto y diciembre de 1988 (Tabla 4).

\section{Análisis comparativo de la comunidad planctónica y epilítica}

La clase Bacillariophyceae contribuyó con un $93 \%$ de las algas en fitoplancton y un $79 \%$ del epiliton. Las muestras de fitoplancton estuvieron formadas además por un $5 \%$ de Chlorophyceae y un $2 \%$ de Cyanophyceae. Las muestras del epiliton estuvieron formadas por un $4 \%$ de Chlorophyceae y un $17 \%$ de Cyanophyceae (Fig. 10).

Cocconeis placentula, con las variedades euglypta y lineata, fueron las que más aportaron a la densidad de fitoplancton, mientras que la contribución más elevada a la densidad epilítica estuvo dada por Cymbella affinis y Synedra ulna. Respecto a las Chlorophyceae las especies Spirogyra sp., Dictyosphaerium pulchellum, Scenedesmus ecornis y Pediastrum boryanum fueron las principales contribuyentes a la densidad de la fitoplancton y Stigeoclonium stagnati-
Tabla 4. Comparación del Indice de Diversidad (Shannon-Weaver) y Equitabilidad de la comunidad fitoplanctónica y epilítica del río Piedra Blanca (Córdoba, Argentina) durante el periodo Junio 1988Junio 1989. Comparison of phytoplankton and epilithon Diversity (Shannon-Weaver) and Equitability Index during the period June 1988-June 1989.

\begin{tabular}{|c|c|c|c|c|}
\hline \multirow{2}{*}{ Meses } & \multicolumn{2}{|c|}{ Diversidad } & \multicolumn{2}{|c|}{ Equitabilidad } \\
\hline & Fitopl. & Epil. & Fitopl. & Epil. \\
\hline Jun 88 & 2.40 & 2.91 & 0.83 & 0.74 \\
\hline Jul & 2.00 & 3.00 & 0.57 & 0.77 \\
\hline Ago & 2.63 & 3.75 & 0.77 & 1.00 \\
\hline Set & 1.63 & 3.45 & 0.52 & 0.93 \\
\hline Oct & 2.58 & 3.92 & 0.69 & 0.98 \\
\hline Nov & 2.72 & 3.90 & 0.67 & 0.87 \\
\hline Dic & 3.57 & 3.79 & 0.79 & 1.00 \\
\hline Ene & 3.36 & 0.48 & 0.75 & 0.17 \\
\hline Feb & 3.42 & 2.27 & 0.85 & 0.54 \\
\hline Mar & 2.67 & 2.99 & 0.77 & 0.78 \\
\hline Abr & 2.94 & 3.40 & 0.84 & 0.98 \\
\hline May & 2.87 & 4.15 & 0.75 & 0.89 \\
\hline Jun 89 & 2.17 & 3.97 & 0.56 & 0.89 \\
\hline
\end{tabular}

le, Monoraphidium sp. y Cladophora glomerata al epiliton. De las Cyanophyceae, Lyngbya sp. fue la especie que contribuyó a la densidad de ambos compartimientos (Tabla 3).

El dendrograma obtenido por la aplicación del método de clasificación divisivo Twinspan organiza dos agrupamientos. El grupo I se conforma de muestras epilíticas, donde la especie asociada más abundante fue Gomphonema minutum. El grupo II se subdivide en el agrupamiento III y IV con asociaciones de muestras de fitoplancton y epilíticas. En estos subgrupos las especies más abundantes fueron: Cocconeis placentula var. euglypta y Navicula cryptocephala en el grupo III y la especie Synedra ulna en el grupo IV (Fig. 11).

\section{Relación de los factores ambientales con los compartimientos fitoplanctónicos y epilíticos}

Entre los factores abióticos analizados, se seleccionaron aquellos que presentaron coeficientes de correlación lineal significativos con alguna variable biológica: temperatura, $\mathrm{pH}$, conductividad y sólidos disueltos. 


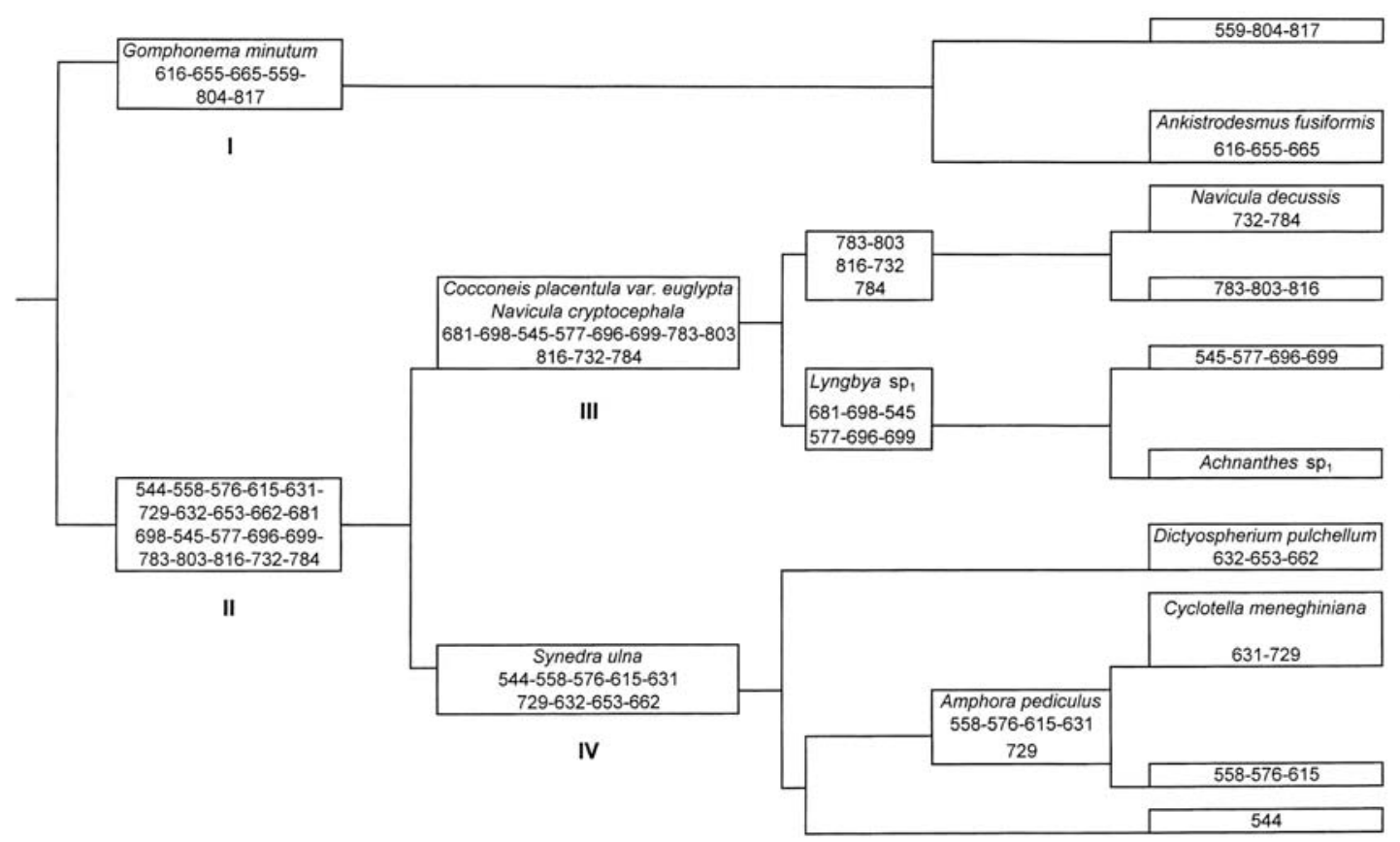

Figura 11. Dendrograma de la clasificación de las especies algales asociadas a las muestras mediante la aplicación del método Twinspann. $\mathrm{N}^{\circ}$ : ver programa de muestreo. Dendrograph showing the classification of algae species associated to the samplings (Twinspan method). $N^{o}$ : see sampling programme.

En la fitoplancton el pH, la conductividad y la concentración de sólidos disueltos fueron los factores que más estrechamente se relacionaron con la riqueza específica total y la riqueza específica de diatomeas en forma positiva y con la diversidad negativamente $(\mathrm{p}<0.01 \mathrm{y}$ $\mathrm{p}<0.05)$. Estos factores también estuvieron relacionados negativamente con la densidad de clorofitas con valores ligeramente menores $(p<0.05)$. La conductividad y los sólidos disueltos se relacionaron negativamente con una de las especies más abundantes Reimeria uniseriata (Tabla 5).

En la comunidad epilítica, el $\mathrm{pH}$, la conductividad y los sólidos disueltos incidieron negativamente en la diversidad y positivamente con la equidad $(\mathrm{p}<0.01)$. De éstos valores la concentración de sólidos disueltos obtuvo relaciones negativas significativas con la densidad de diatomeas y con la densidad de clorofitas $(\mathrm{p}<0.01)$. Algunas de las especies más abun- dantes de diatomeas, tales como Cocconeis placentula, Gomphonema parvulum y Achnanthes minutissima, se relacionaron negativamente con el pH y la conductividad ( $\mathrm{p}<0.01$ ), mientras que Navicula cryptocephala y Reimeria uniseriata solo se relacionaron con la conductividad $(\mathrm{p}<0.01)$ (Tabla 5).

\section{DISCUSIÓN}

La comunidad algal del río Piedra Blanca presentó características que, en general, no se apartan de las dadas a conocer en otros ríos de ambientes serranos. Las especies algales dominantes en los compartimientos de fitoplancton $\mathrm{y}$ adherido pertenecieron a la clase Bacillariophyceae y Chlorophyceae, componentes que se reportan para ríos y arroyos del país y otras latitudes (Esho \& Benson-Evans, 1984, Esho \& Benson-Evans, 1984, Martínez de Fabricius, 
Tabla 5. Coeficiente de correlación lineal simple (r), múltiple $(\mathrm{R})$ y de determinación simple $\left(\mathrm{r}^{2}\right)$ y múltiple $\left(\mathrm{R}^{2}\right)$. Variable dependiente -cada descriptor biológico (1)-; variable independiente -factores abióticos. Referencias: 2: temperatura; 3: pH; 4: conductividad; 5: sólidos disueltos. Coefficients of simple $(r)$ and multiple $(R)$ lineal correlation, and of simple $\left(r^{2}\right)$ and multiple determination $\left(R^{2}\right)$. References: 2: temperature; 3: $\mathrm{pH}$; 4: conductivity; 5 : dissolved solids.

\begin{tabular}{|c|c|c|c|c|c|c|c|c|}
\hline FITOPLANCTON & $\mathbf{R}_{1,2}$ & $\mathbf{r}_{1,2}^{2}$ & $\mathbf{R}_{1,23}$ & $\mathbf{R}_{1,23}^{2}$ & $\mathbf{R}_{1,234}$ & $\mathbf{R}_{1,234}^{2}$ & $\mathbf{R}_{1,2345}$ & $\mathbf{R}_{1,2345}^{2}$ \\
\hline Densidad & -0.2398 & 5.75 & 0.2398 & 5.75 & 0.4098 & 16.80 & 0.5244 & 27.50 \\
\hline Densidad diatomeas & -0.2184 & 4.77 & 0.2235 & 4.99 & 0.4183 & 17.49 & 0.4508 & 20.32 \\
\hline Densidad Chlorophyta & -0.4840 & 23.42 & 0.5630 & 31.70 & 0.5720 & 32.71 & 0.6500 & 42.26 \\
\hline Riqueza específica (RE) & 0.4851 & 23.53 & 0.6594 & 43.48 & 0.6676 & 44.56 & 0.7220 & 52.12 \\
\hline R E diatomeas & 0.3929 & 15.44 & 0.6463 & 41.77 & 0.6515 & 42.45 & 0.6992 & 48.89 \\
\hline Diversidad & -0.5112 & 26.13 & 0.7401 & 54.78 & 0.7522 & 56.58 & 0.7563 & 57.19 \\
\hline Equitabilidad & -0.2296 & 5.27 & 0.1294 & 1.67 & 0.3298 & 10.87 & 0.4445 & 19.76 \\
\hline Cocconeis placentula & -0.1333 & 1.78 & 0.3159 & 9.98 & 0.4172 & 17.41 & 0.4946 & 24.46 \\
\hline Cymbella affinis & -0.0053 & 0.00 & 0.2989 & 8.93 & 0.3913 & 15.31 & 0.4374 & 19.13 \\
\hline Reimeria uniseriata & -0.2320 & 5.38 & 0.2716 & 7.38 & 0.5394 & 29.09 & 0.5980 & 35.76 \\
\hline Synedra ulna & 0.3832 & 14.68 & 0.4777 & 22.82 & 0.5062 & 25.62 & 0.5298 & 28.07 \\
\hline EPILITON & $\mathbf{R}_{1,2}$ & $\mathbf{r}_{1,2}^{2}$ & $\mathbf{R}_{1,23}$ & $\mathbf{R}_{1,23}^{2}$ & $\mathbf{R}_{1,234}$ & $\mathbf{R}_{1,234}^{2}$ & $\mathbf{R}_{1,2345}$ & $\mathbf{R}^{2}{ }_{1,2345}$ \\
\hline Densidad & -0.2600 & 6.76 & 0.3089 & 9.54 & 0.3888 & 15.12 & 0.4871 & 23.72 \\
\hline Densidad diatomeas & -0.4002 & 16.02 & 0.4571 & 20.89 & 0.5213 & 27.17 & 0.7022 & 49.31 \\
\hline Densidad Chlorophyta & 0.2060 & 4.25 & 0.5377 & 28.91 & 0.5562 & 30.93 & 0.6644 & 44.15 \\
\hline Riqueza específica (RE) & -0.0373 & 0.14 & 0.2644 & 6.99 & 0.3925 & 15.40 & 0.4908 & 24.09 \\
\hline R E diatomeas & -0.1581 & 2.50 & 0.3701 & 13.70 & 0.4929 & 24.29 & 0.5263 & 27.70 \\
\hline Diversidad & -0.2245 & 5.04 & 0.7107 & 50.50 & 0.7107 & 50.51 & 0.7261 & 52.73 \\
\hline Equitabilidad & 0.2478 & 6.14 & 0.7146 & 51.06 & 0.7163 & 51.30 & 0.7163 & 51.31 \\
\hline Cocconeis placentula & -0.6139 & 37.69 & 0.6502 & 42.28 & 0.8121 & 65.94 & 0.8579 & 73.60 \\
\hline Gomphonema parvulum & -0.5836 & 34.06 & 0.7454 & 55.56 & 0.7532 & 56.73 & 0.7597 & 57.72 \\
\hline Navicula cryptocephala & -0.5108 & 26.09 & 0.5175 & 26.78 & 0.8984 & 80.71 & 0.9284 & 86.19 \\
\hline Reimeria uniseriata & -0.5647 & 31.89 & 0.5774 & 33.34 & 0.7157 & 51.22 & 0.8031 & 64.50 \\
\hline Achnanthes minutissima & -0.6390 & 40.84 & 0.7037 & 49.52 & 0.7418 & 55.03 & 0.8689 & 75.50 \\
\hline
\end{tabular}

1986; Martínez de Fabricius \& Corigliano, 1989; Zalocar de Domitrovic, 1992; O'Farrell, 1993; Gosselain et al., 1994, Rojo et al., 1994, Anselmi de Manavella \& García de Emiliani, 1995). Las Cyanophyceae sólo predominaron en el epiliton, el resto de las clases algales estuvieron pobremente representadas durante el período de estudio.

Los efectos de la velocidad de corriente sobre la abundancia del epiliton han sido mencionados por diversos autores (Ács \& Kiss, 1993; Stevenson \& Glover, 1993). Peterson \& Stevenson (1992) afirmaron que tanto la estructura de la comunidad así como la diversidad están periódicamente sujetas a las variaciones de la velocidad de corriente, e incluso están aso- ciadas a la presencia de hábitats secundarios representados por algas filamentosas. En relación a este factor Antoine \& Benson-Evans (1982) comprobaron que cuando las variaciones de la velocidad de corriente son importantes se produce una relación inversa entre la densidad de la comunidad epilítica y esta variable. Especies caracterizadas por Patrick (1977) y Kawamura \& Hirano (1992) por presentar hábito bentónico se han encontrado únicamente formando parte del fitoplancton. Esta acentuada proporción de organismos "no planctónicos" en la fracción de fitoplancton hace suponer un aporte más o menos contínuo del epiliton como consecuencia de las características geomorfológicas e hidrológicas del río. 
Una particularidad de esta última comunidad fue su elevada densidad en relación a otros ríos de la provincia de Córdoba de similares características (Martínez de Fabricius \& Corigliano, 1989; Luque et al., 1997, Luque \& Martínez de Fabricius, 2000). La máxima riqueza de especies y densidad se corresponde inversamente con las precipitaciones: cuando los valores pluviométricos son máximos, la densidad del epiliton disminuye abruptamente. Esto coincide con lo puntualizado por Hynes (1970) y Liaw \& McCrimmon (1978) que consideran que en un río de régimen torrencial, como el que presenta el río Piedra Blanca, la acción abrasiva de la corriente es un factor responsable de la pérdida de algas epilíticas.

Las Bacillariophyceae predominaron cualitativamente y cuantitativamente en ambas comunidades, principalmente el orden Pennales, mientras que especies del orden Centrales estuvieron poco representadas. Estos resultados difieren de las observaciones de Molloy (1992) quien afirma que las diatomeas céntricas se presentan con mayor frecuencia río abajo y no en tramos de cabecera. Sin embargo, investigaciones realizadas en el río Cuarto por Martínez de Fabricius (1996) ponen en evidencia que el déficit de diatomeas céntricas ocurre tanto en zonas de cabeceras como río abajo.

En el río Piedra Blanca, las condiciones ambientales, alta transparencia del agua, moderada velocidad de corriente, poca profundidad, largos períodos de luz solar y temperaturas relativamente altas, favorecen el desarrollo de clorófitas, especialmente filamentosas, las que predominaron en número de especies y densidad durante los meses de primavera-verano. Resultados similares se obtuvieron para otros cursos de agua bajo condiciones hidrológicas semejantes (Martínez de Fabricius, 1986; Martínez de Fabricius \& Corigliano, 1989; O'Farrell, 1993). El número de especies de Desmidiaceae, y su mayor frecuencia en la fitoplancton se corresponde con las condiciones físicas y químicas señaladas como adecuadas para su ocurrencia, mientras que en las épocas de estiaje disminuye, coincidente con un aumento de la concentración iónica, elevados valores de conductividad y sólidos disueltos. La relación negati- va de las Desmidiáceas con la conductividad, $\mathrm{pH}$ y sólidos disueltos coinciden con los estudios realizados por O'Farrell \& Izaguirre (1994) y O'Farrell (1994) en las cuenca del Río de La Plata.

Es muy frecuente observar filamentos de Cladophora glomerata en los meses de septiembre y octubre época en la cual esta macroalga presenta su máximo crecimiento. Parte del talo se desprende y es transportado por la corriente (Martínez de Fabricius, 1986; Dell'uomo, 1991). Cabe señalar la presencia ocasional de Stigeoclonium stagnatile con elevada densidad en el mes de junio de 1989. Estudios realizados por Antoine \& Benson-Evans (1985) afirman que es más frecuente encontrar la porción postrada de dicha especie. En el río Piedra Blanca, en cambio, se presentó con mayor frecuencia el talo completo de esta especie, no fragmentado en su porción postrada y erecta.

La dominancia de las Cyanophyceae en el epiliton durante el verano también se ha comprobado en estudios realizados en el río Samborombón por Solari \& Claps (1996).

La estructura y dinámica de la comunidad de fitoplancton y epilítica del río Piedra Blanca, estuvieron controladas por factores físicos interactuando con los hidrológicos. Esta marcada influencia de los factores físicos, se ha señalado en la literatura para otros sistemas lóticos (Reynolds, 1992; Anselmi de Manavella \& García de Emiliani, 1995).

\section{CONCLUSIONES}

- La comunidad fitoplanctónica fue la que registró mayor riqueza específica, mientras que la epilítica predominó en densidad de organismos.

- La clase Bacillariophyceae predominó sobre las restantes clases algales, destacándose las diatomeas pennadas.

- Las Chlorophyceae fueron subdominantes en el fitoplancton, en el epiliton subdominaron las Cyanophyceae.

- El pH, conductividad y sólidos disueltos fueron los factores abióticos que mejor explica- 
ron las variaciones de los descriptores biológicos en ambas comunidades.

- El método Twispan separó en primer instancia las muestras según la densidad de una determinada especie mientras que los agrupamientos finales se subdividieron según las comunidades a la cual pertenecen las muestras.

\section{BIBLIOGRAFÍA}

ÁCS, É. \& K. T. KISS. 1993. Effects of the water discharge on periphyton abundance and diversity in a large river (River Danube, Hungary). Hydrobiología, 249: 125-133.

ALLAN, J. D. 1995. Stream Ecology. Structure and function of running waters. Chapman \& Hall Edts. N. Y.

ANSELMI de MANAVELLA, M. I. \& M. O. GARCÍA de EMILIANI. 1995. Composición y dinámica del fitoplancton en una sección transversal del río Correntoso (Llanura aluvial del Río Paraná). Rev. Asoc. Cs. Nat. Litoral, 26 (2): 39-54.

ANTOINE, S. E. \& K. BENSON-EVANS. 1982. The effect of current velocity on the rate of growth of benthic algal communities. Int. Rev. Ges. Hydrobiol., 67: 575-583.

ANTOINE, S. E. \& K. BENSON-EVANS. 1985. Benthic algal flora of the River Wye System, Wales, U.K. Nova Hedwigia, 42: 31-47.

ARCHIBALD, R. E. M. 1983. The Diatoms of the Sundays and Great at Fish Rivers in the Eastern Cape Province of South Africa. J. Cramer Vaduz. Bibl. Diatomológica, 1: 1-362, 34 láms.

BOURRELLY, P. 1981. Les Algues d'eau douce. Les algues jaunes et brune. II. Ed. Boubée, París.

BOURRELLY, P. 1985 Les Algues d'eau douce. Les algues bleues et rouges. III. Ed. Boubée, París.

BOURRELLY, P. 1990. Les Algues d'eau douce. Les algues vertes. I. Ed.Boubée, París.

CONFORTI, V., J. ALBERGHINA \& E. GONZÁLEZ URDA. 1995. Structural changes and dynamics of the phytoplankton along a highly polluted lowland river of Argentina. J. Aquatic Ecosystem Health, 4: 59-75.

CORIGLIANO, M. del C., A. L. MARTÍNEZ de FABRICIUS, M. E. LUQUE \& N. GARI. 1994. Patrones de distribución de variables fisicoquímicas y biológicas en el río Chocancharava (Cuarto) (Córdoba, Argentina). Rev. UNRC, 14(2): 177-194.
DEL GIORGIO, P. A., A. L. VINOCUR, R. J. LOMBARDO \& G. TELL. 1991. Progressive changes in the structure and dynamics of the phytoplankton community along a pollution gradient in a lowland - a multivariate approach. Hydrobiologia, 224: $129-154$.

DELL'UOMO, A. 1991. Use of benthic macroalga for monitoring rivers in Italy. En: Use of algae for monitoring rivers. B. A. Whitton, E. Rott \& G. F. Friedrich (eds.): 129-137. Institut für Botanik, Universität Innsbruck.

DESIKACHARY, T. V. 1959. Cyanophyta. New Delhi. Ed. Board. $545 \mathrm{pp}$.

ESHO, R. T. \& K. BENSON-EVANS. 1984. Algal studies of the River Ely, South Wales, U. K. II Epilithic Algae. Nova Hedwigia, 40: 387-421.

ESHO, R. T. \& K. BENSON-EVANS. 1984. Algal studies of the River Ely, South Wales, U. K. I. Phytoplankton. Nova Hedwigia, 40: 347-368.

GARCÍA de EMILIANI, M. O. 1990. Phytoplankton ecology of the Middle Paraná River. Acta Limnol. Brasil, 3: 391-417.

GERMAIN, H. 1981. Flore des Diatomeées. Paris. Ed. Boubée. 448 pp.

GOSSELAIN, V.; J. P. DESCY \& E. EVERBECQ. 1994. The phytoplankton community of the River Meuse, Belgium: seasonal dynamic (year 1992) and the possible incidence of zooplankton grazing. En:. Phytoplankton in turbid environments: river and shallow lakes. J. P. Descy, C. S. Reynolds \& J. Padisák (eds.). Hydrobiología, 289: 179-191.

HILL, M. O. 1979. TWINSPAN-a FORTRAN program for arranging multivariate data in an ordered twoway table by classification of the individuals and attributes. Ecology and Systematics, Cornell University, Ithaca, N. Y.

HYNES, H. B. N. 1970. The Ecology of Running Waters. Univ. Toronto Press., Toronto.

KAWAMURA, A. \& R. HIRANO. 1992. Seasonal changes in benthic diatom commmunities colonizing glass slides in Aburatsubo bay, Japan. Diat. Res. 7(2): 227-239.

KRAMMER, K. \& H. LANGE-BERTALOT. 1986. Bacillariophyceae 2. Naviculaceae. 2/1. Jena. G. F. Verlag. 875 pp.

KRAMMER, K. \& H. LANGE-BERTALOT. 1988. Bacillariophyceae 2. Bacillariaceae, Epithemiaceae, Surirellaceae 2/2. G. Fischer. Stuttgart. 596 pp.

KRAMMER, K. \& H. LANGE-BERTALOT. 1991a. Bacillariophyceae 3. Centrales, Fragilariaceae, 
Eunotiaceae 2/3. New York. G. Fischer. Stuttgart. 576 pp.

KRAMMER, K. \& H. LANGE-BERTALOT. 1991b. Bacillariophyceae 4. Achnanthaceae 2/4. New York. G. Fischer. Stuttgart. 437 pp.

LIAW, W. K. \& H. R. MacCRIMMON. 1978. Assessing changes in biomass of river bed periphyton. Int. Rev. Ges. Hydrobiol., 63: 155-171.

LOEZ, C. R. \& A. SALIBIAN. 1990. Premiéres données sur le phytoplancton et les caractéristiques physico-chimiques du rio Reconquista (Buenos Aires, Argentine). Rev. Hydrobiol. Trop., 23(4): 283-296.

LUQUE, M. E., A. L. MARTÍNEZ de FABRICIUS \& E. N. GARI. 1994. El componente algal en transporte en ríos y arroyos serranos de la cuenca del río Cuarto (Córdoba, Argentina). Tankay, I: 55-57.

LUQUE, M. E., N. GARI \& A. L. MARTÍNEZ de FABRICIUS. 1997. Análisis cualitativo-cuantitativo de la flora algal en el tramo superior de la cuenca del río Chocancharava (ex Cuarto) (Córdoba, Argentina). Rev. UNRC, 17(1): 49-67.

LUQUE, M. E. \& A. L. MARTÍNEZ de FABRICIUS. 2000. Ficoflora fitoplanctónica y epilítica del río Piedra Blanca (Córdoba, Argentina). Bol. Soc. Argent. Bot. 35 (1-2): 21-32.

MARTÍNEZ de FABRICIUS, A. L. 1986. La ficoflora del Río Grande (Departamento de Calamuchita, provincia de Córdoba-Argentina). Rev. UNRC, 6(2): 221-235.

MARTÍNEZ de FABRICIUS, A. L. 1995. Bacillariophycae del Río Cuarto (Córdoba). Nuevas o raras para la Argentina. Bol. Soc. Argent. Bot., 31(1-2): 41-47.

MARTÍNEZ de FABRICIUS, A. L. 1996. Bacillariophycae del Río Cuarto (Córdoba). Tesis Doctoral $\mathrm{N}^{\circ} 673$, Universidad Nacional de La Plata. 298 pp. 17 láms.

MARTÍNEZ de FABRICIUS, A. L., M. C. FERNANDEZ BELMONTE, E. N. GARI \& M. del C. CORIGLIANO. 1988. Análisis del componente algal en transporte en ríos y arroyos del Valle de Calamuchita (Córdoba, Argentina). Rev. UNRC, 8(1): 95-110.

MARTÍNEZ de FABRICIUS, A. L. \& M. del C. CORIGLIANO. 1989. Composición y distribución de comunidades algales en el río Ctalamochita (Córdoba, Argentina). Rev. UNRC, 9(1): 5-13.

MARTÍNEZ de FABRICIUS, A. L. \& E. N. GARI. 1989. Estudios sistemáticos de las bacillariophycae del Río Grande (Dpto. Calamuchita, Prov. Córdoba,
Argentina), Pennales I. Bol. Soc. Argent. Bot., 26(1): 13-21.

MARTÍNEZ de FABRICIUS, A. L. \& E. N. GARI. 1991. Estudios sistemáticos de las Bacillariophycae del Río Grande (Dpto. Calamuchita, Prov. Córdoba, Argentina), Pennales II. Bol. Soc. Argent. Bot., 27(3-4): 207-215.

MARTÍNEZ DE FABRICIUS, A. L., GARI, E. N. 1996. Estudios Sistemáticos de las Bacillariophycae del Río Grande (Departamento de Calamuchita, Córdoba, Argentina). Pennales III. Bol. Soc. Argent. Bot., 32(1-2): 21-35.

MOLLOY, J. M., 1992. Diatom communities along stream longitudinal gradients. Freshwater Biol., 28: 59-69.

O'FARRELL, I. 1993. Phytoplankton ecology and limnology of the Salado River (Buenos Aires, Argentina). Hydrobiología, 271: 169-178.

O'FARRELL, I. \& I. IZAGUIRRE. 1994. Phytoplankton ecology and limnology of the River Uruguay Lower Basin. (Argentina). Arch. Hydrobiol. Suppl., 99(1/2): 155-179.

O'FARRELL, I. 1994. Comparative analysis of the phytoplankton of fifteen lowland fluvial systems of the River Plate Basin (Argentina). Hydrobiología, 289: 109-117.

PATRICK, R. \& C. W. REIMER. 1966. The Diatoms of the United States exclusive of Alaska and Hawai. Vol. 1. Acad. Nat. Sci. Philadelphia. Monog., 13: 1688.

PATRICK, R. \& C. W. REIMER. 1975. The Diatoms of the United States exclusive of Alaska and Hawai. Vol. 2. Acad. Nat. Sci. Philadelphia. Monog., 13: 1213.

PATRICK, R. 1977. Ecology of freshwater diatoms and diatom communities (p. 284-332). En: D. Werner (Ed.). The Biology of diatoms. Oxford. London. (Bllackkwell Scientific publications). $498 \mathrm{pp}$.

PETERSON, C. G. \& R. J. STEVENSON. 1992. Resistance and resilience of lotic algal communities: importance of disturbance timing and current. Ecology, 73 (4): 1445-1461.

PRESCOTT, G. M. 1962. Algae of the Western Great lake area .N. M. C. Brow. Co. Ine. 660 pp.

REYNOLDS, C. S. 1992. Algae. En: The Rivers Handbook. P. Calow \& G. E. Petts (eds.) Vol. 1: 195-215.

ROUT, J. \& J. P. GAUR. 1994. Composition and dynamics of epilithic algae in a forest stream at Shillong (India). Hydrobiologia, 291: 61-74. 
SABATER, S., F. SABATER \& J. ARMENGOL. 1988. Relationships between Diatom Assemblages and Physico-chemical variables in the River Ter (N. E. Spain). Int. Revue ges. Hydrobiol., 73: 171179.

SHANNON, C. E. \& W. WEAVER. 1963. The mathematical theory of communication. Univ. Illiniois Press, Urbana.

SINSABAUGH, R. L., T. WEILAND \& A. E. LINKINS. 1991. Epilithon patch strucutre in boreal river. J. N. Am. Benthol. Soc., 10 (4): 419-429.

SIMONSEN, R. 1979. The Diatom System. Ideas on Phylogeny. Bacillaria, 2: 9-71.

SOKAL, R. \& F. ROHLF. 1979. Biometría. Blume, Madrid.

SOLARI, L. C. 1995. Structure and dynamics of phytoplankton of the River Samborombón (Buenos Aires, Argentina). Acta Hydrobiol., 37(4): 231-241.

SOLARI, L. C. \& M. C. CLAPS. 1996. Planktonic and benthic algae of a pampean river (Argentina): comparative analysis. Annals limnol. 32(2): 89-95.

STEVENSON R. J. \& R. GLOVER. 1993. Effects of algal density and current on ion transport through periphyton communities. Limnol. Oceanogr., 38(6): 1276-1281.

STRAHLER, A. N. 1981. Geografía Física. Ed. Omega. Barcelona.

VENRICK, E. L. 1995. Muestreo y submuestreo del fitoplancton marino y dulceacuícola. En: Manual de Métodos Ficológicos. E. C. Oliveira \& E. Sar (eds.): 200-210.

VILLAFAÑE, V. E. \& F. M. H. REID. 1995. Métodos de microscopia para la cuantificación del fitoplancton. En: Manual de Métodos Ficológicos. K. Alveal, M. E. Ferrario, E. C. Oliveira \& E. Sar (eds): 169-185.

ZALOCAR de DOMITROVIC, Y. 1992. Fitoplancton de ambientes inundables de río Paraná (Argentina). Rev. Hydrobiol. Trop., 25(3): 177-188. 